\title{
Target-specific airborne geophysical forecast-exploration technology for diamond deposits «Field - Cluster - Pipe»
}

\section{A. Tsyganov, R. S. Kontarovich}

State Scientific - Production Enterprise «AeroGeoPhysica», 4, Nizhnyaya Krasnoselskaya ul, Moscow, 107140 Russia,

The current level of airborne geophysics enables changing the technological use of airborne geophysics to solve different applied geological problems.

Instead of traditional direct prospection for diamond deposits, using local geophysical anomalies, and occasional, irregular attempts to identify other forecast factors, the current state of geophysics allows the development and use of target-oriented airborne-geophysical forecast-exploration technologies, designed to effectively discover specific commercial types of diamond deposits in specific landscapegeological environments.

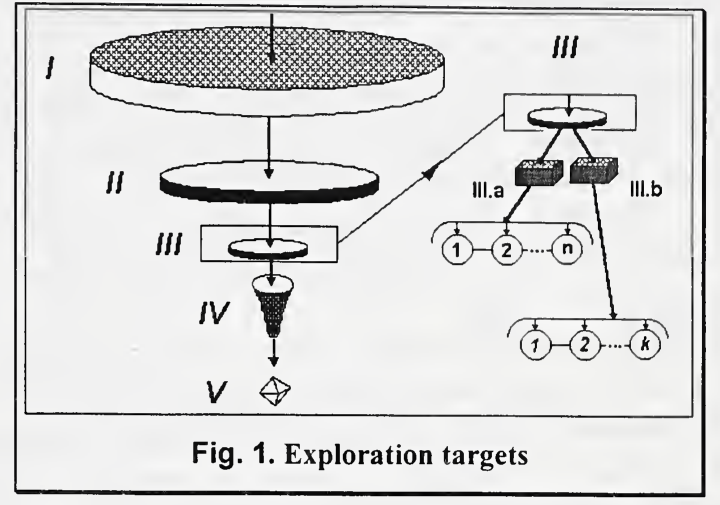

The said circumstances encourage the development of standard, target-oriented airbornegeophysical-geological research technologe fully designed to resolve specific applied problems in the most efficient way. In each particular instance, the technology focus must be supported with -

- target-specific choice of required work stages, airborne geophysical methods and modifications, measurement accuracy, survey scales and flight altitudes for different work stages,

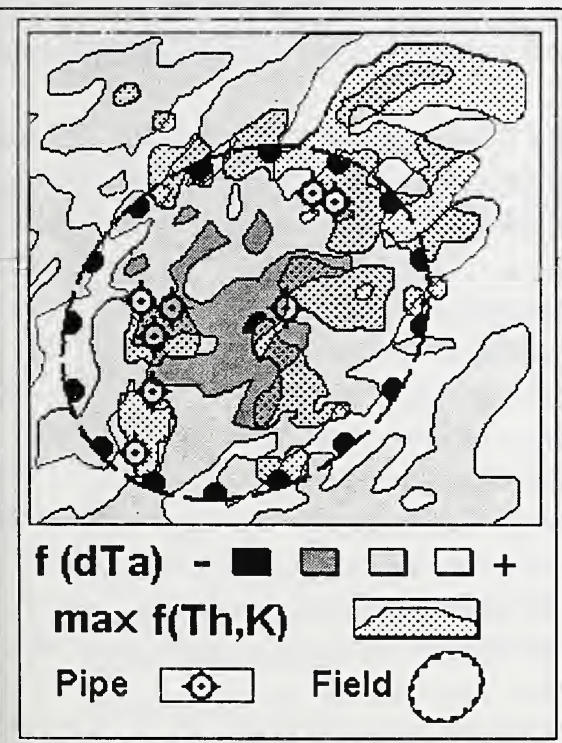

Fig. 2 Small-scale airborne magnetics and gamma-ray surveys - kimberlite field
- target-specific methods of data processing and presentation, a set of required additional information,

- target-specific flowchart for general and forecast data interpretation.

Let's discuss and illustrate the general principles of development of target-specific airborne-geophysical forecast-exploration technologies for diamonds, with exploration applications as an example.

Exploration targets. In the first approximation, a profitable diamond deposit in specific geo-economical conditions is the target of any exploration. On second thought, however, one can see that among the targets are a great number of explorationally important geological phenomena, and their logical identification aggregately determines the work technology.

Fig. 1 shows a part of logical identification of an exploration target in diamond primary deposit prospecting. Index I indicates an exploration target to be distinguished during the early exploration stage - the "mineragenic zone". Within a diamond-bearing province and subprovince it is linear or 
near-linear geological structure accommodating all known kimberlite fields. Hence, during diamond prospecting in sufficiently large areas, the diamond mineragenetic zone is an independent exploration target. After identifying a prospective diamondbearing mineragenic zone, the step is to delimit it within areas prospective for kimberlite field discovery (Index II in Figure 1). The next step is to detect within these areas local sites prospective for kimberlite body clusters (Index III in Fig. 1) and, within them, detailed areas and anomalies prospective for kimberlite pipes (Index IV in Fig. 1).

In the given example, the threshold-profitable deposit (see $\mathbf{V}$ in Fig. 1) serves as an end exploration target, and the mineragenic zone, field, cluster, pipe as intermediate targets.. All the exploration targets (in our case, a diamond-bearing province - subprovince - diamond-bearing mineragenic zone - kimberlite field - pipe cluster - pipe) form $a$ hierarchical series of intermediate exploration targets.

Intermediate targets admit of two work flowcharts or combinations thereof in practical exploration. These are the above-described phased research and reduced exploration which makes it possible, under favorable conditions (contrasted indicatory features of exploration targets, favorable landscape-geological environment), to skip some work stages. It is important to note that a reduced technology

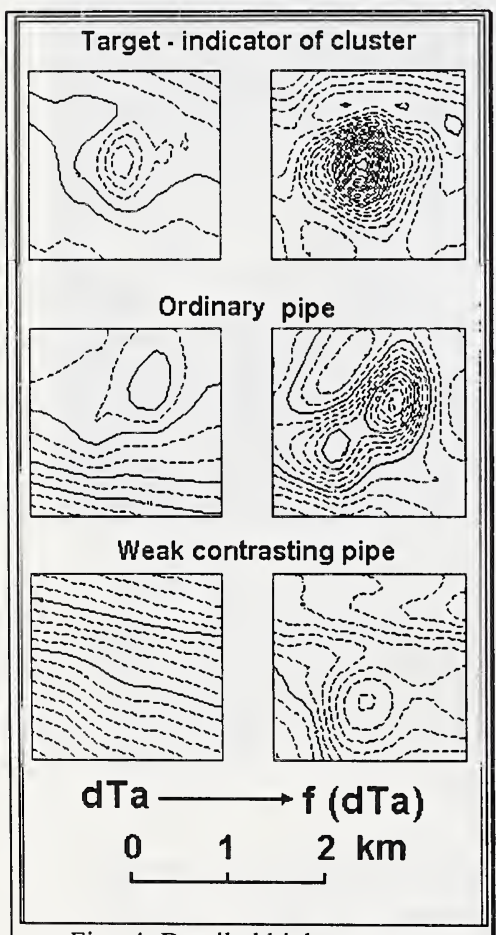

Fig. 4. Detailed high-accuracy airborne magnetometer surveys is less reliable and, as a rule, is replaced by a phased approach in due

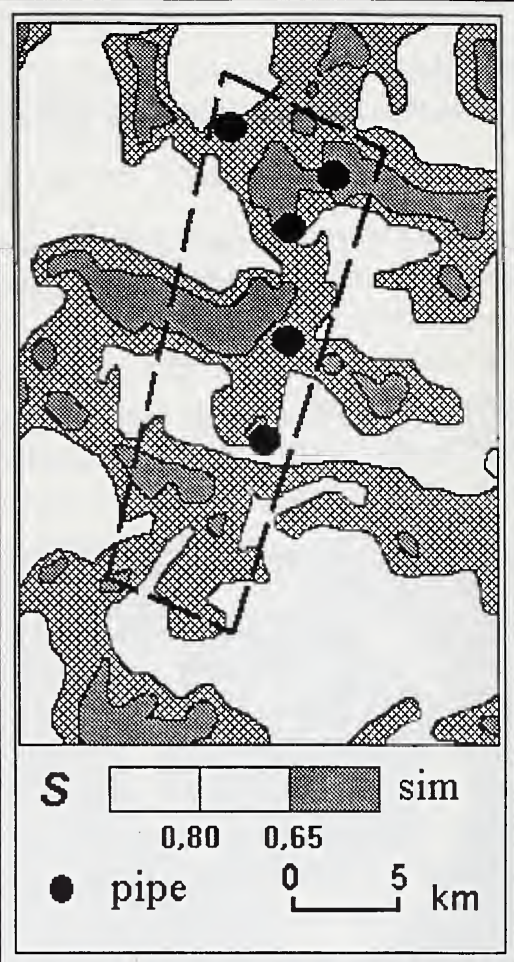

Fig. 3. Medium-scale airborne EM, surveys - kimberlite claster course.

The right-hand part of Figure 1 is a schematic representation of the technology of identifying an intermediate target, using the method of identifying kimberlite-cluster prospective sites (Index III) in the way of example. Index IIIa shows geological phenomena reflecting geological regularities in the development of the given territory, which account for the specific location of the respective member of exploration target hierarchy (exploration prerequisites); IIIb is geological phenomena reflecting peculiar features of the exploration target, expressed in structural peculiarities of the host landscape-geological medium (exploration indications). Hence, both exploration prerequisites and exploration indications, while they are most important factors of exploration target (intermediate) localization, also turn out to be exploration targets. They can be called particular exploration targets.

Exploration prerequisites used in exploration are quite diverse. They are geological regularities manifesting themselves at various material levels of terrain structure: geochemical, 
mineralogical, petrographical (or lithological), formational, and tectonical $(\mathbf{1}, \mathbf{2}, \ldots, \mathbf{n}$ in Fig. 1). The manifestation forms of exploration indications - geological phenomena indicating (directly or indirectly) the presence of an intermediate exploration target or, to be more precise, the fact of its formation, are rather diverse, too $(1,2, \ldots . ., k$ in Fig. 1). They appear in the same particular geological fields or static geological systems.

The important feature of static geological systems is material reflection of regularities typical of systems holding a higher hierarchic position (for example, formational or tectonic), in systems of a relatively low level (mineralogical or geochemical). Consequently, almost all exploration prerequisites and indications can be clearly reflected in the simplest material geological fields: geochemical, mineralogical, petrographical.

The variability of geological fields, by its nature, reflects, to various extents, the variability of simple geological fields, and with them, with a different degree of contrast, exploration prerequisites and indications.

With this approach, every metrically reliable

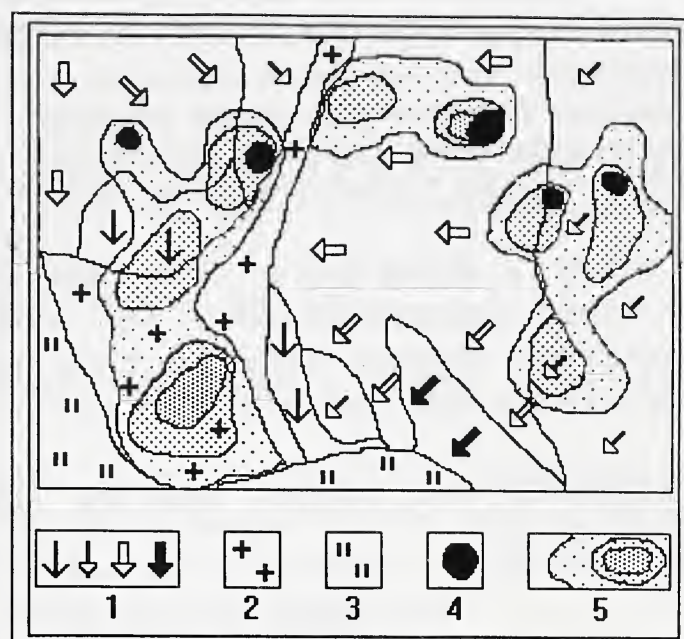

Fig. 5 Detailed high-accuracy airborne gamma-ray survey

1-slopes of various stepness, 2-foot of slopes, 3-flood plain of a river, 4- kimberlite pipes, 5- secondary radiogeochemical halos of kimberlite pipes geological phenomenon, manifesting itself in one form or another in measured or described geological-geophysical fields, must have a semantic classification label in geological or landscape-geological terms. Between these phenomena, interrelations are established, like "higher-lower", "younger-older", "bedrock-landscape", and so on. By this way, geological and landscape-geological maps of territories are created.

The last data interpretation step is the transition from geological and landscape-geological models to logical-semantic target-specific models. All geological phenomena described in the course of interpretation and unexplained elements of geophysical fields are then classified into the some forecast-exploration categories:

1) Prerequisites of explorational forecasting of a single or several exploration target out of the preset intermediate exploration targets;

2) 2)Direct or indirect indications of a single or several exploration targets out of the pre-set intermediate exploration targets;

3) 3) Phenomena complicating identification of concrete particular exploration targets ;

4) 4) Phenomena which do not have a forecast value and do not influence identification of data which is useful for the purpose. Next, a forecast map is made for the territory of work, with a spatial picture of identified prerequisites and indications of intermediate exploration targets, i.e. a forecast map of the respective work stage.

For each of the identified forecast factors, an area model of assessing the reliability of identifying that factor is then made, and on the basis of a group of models a complete map of exploration reliability assessment for the territory. 